\section{Reply to Speckman and Rouder: A theoretical basis for QML}

\author{
ANDREW HEATHCOTE \\ University of Newcastle, Newcastle, New South Wales, \\ Australia \\ and \\ SCOTT BROWN \\ University of California, Irvine, California
}

Speckman and Rouder (2004) point out that Heathcote, Brown, and Mewhort (2002) did not prove that their quantile-based estimator (quantile maximum likelihood, or QML) approximates likelihood, but note that it outperformed an exact maximum likelihood method based on order statistics for small samples $(n=20)$ from the exGaussian distribution. When QML uses all order statistics as quantile estimates, it is equivalent to maximum spacings product (MSP) estimation (Cheng \& Amin, 1983; Ranneby, 1984), which can be derived from information theory as a measure of model fit (Ekström, 2001). MSP has been shown to have asymptotic estimation properties that closely parallel conventional maximum likelihood (CML) for regular problems, and better performance in irregular problems, such as estimation of heavy-tailed distributions, mixtures of continuous distributions, and distributions with a minimum that must be estimated (shift distributions). CML can produce inconsistent estimates under these conditions, in particular for shift distributions commonly used in response time research, such as the lognormal, gamma, and Weibull, whereas MSP maintains efficiency or is "hyperefficient."1

MSP is apparently obscure; neither we nor the reviewers of our earlier work were aware of MSP or its link to QML. We became aware of MSP in research for a paper on estimation software for shift distributions (Heathcote, Brown, \& Cousineau, 2004). QML generalizes MSP by using linear combinations of order statistics (i.e., empirical quantile estimates). Titterington (1985) suggested a similar extension of MSP that uses averages of adjacent order statistics and is equivalent to the QML1 method that Heathcote et al. (2002) found to provide the best performance among the special cases of QML they examined.

Cheng and Iles (1987) point out that like MSP, Titterington's (1985) method (and hence QML1) is consistent in irregular problems, and they comment that it is a "viable alternative [to MSP], though we have yet to investigate details" (p. 99). We performed a preliminary investigation of these details by comparing MSP to QML1 estimates using Speckman and Rouder's (2004) simula-

Correspondence should be addressed to A. Heathcote, School of Behavioural Sciences, Aviation Building, University of Newcastle, University Avenue, Callaghan, 2308 NSW, Australia (e-mail: andrew.heathcote@newcastle.edu.au). tion set-up, but with 20,000 replicates. Both MSP and QML1 estimates were obtained by minimizing

$$
\sum_{j=2}^{m} \ln \left(D_{j}\right), D_{j}=\int_{\hat{q}_{j-1}}^{\hat{q}_{j}} f(t, \boldsymbol{\theta}) d t,
$$

where $f(t, \boldsymbol{\theta})$ is the ex-Gaussian density at $t$ and $\boldsymbol{\theta}=(\mu$, $\sigma, \tau)$. For MSP, $m=n+2$ and $\hat{\mathbf{q}}=\left(-\infty, x_{(1)}, x_{(2)}, \ldots\right.$, $\left.x_{(n)}, \infty\right)$. For QML1, $\hat{\mathbf{q}}=\left(-\infty,\left(x_{(1)}+x_{(2)}\right) / 2,\left(x_{(2)}+\right.\right.$ $\left.\left.x_{(3)}\right) / 2, \ldots,\left(x_{(n-1)}+x_{(n)}\right) / 2, \infty\right)$ and $m=n+1$. In both cases, $x_{(i)}$ is the $i$ th order statistic of the sample. Both methods performed similarly, with MSP slightly less biased (for MSP/QML1, mean bias of $\mu$ was 1.8/8.3, for $\sigma$ it was $2.8 /-1.1$, and for $\tau$ it was $4.3 /-5.9)$, but QML1 was slightly more efficient $[S D(\hat{\mu}), 36.4 / 35.4 ; S D(\hat{\sigma})$, 27.1/25.3; $S D(\hat{\tau}), 44.5 / 41.2]$.

We conclude that QML1 is a viable alternative to MSP and that researchers can take advantage of the superior estimation properties of QML1 assured of consistency even in irregular cases. More work is needed to derive results for the efficiency of QML1, and for the consistency, efficiency, and asymptotic distribution of QML estimates based on alternative sets of quantiles (see Brown \& Heathcote, 2003, for results related to asymptotic distribution). ${ }^{2}$ However, we agree with Speckman and Rouder (2004) that QML does not have an exact theoretical basis in likelihood (although it often approximates likelihood) and might better be named quantile maximum probability product (QMP) estimation.

\section{REFERENCES}

Brown, S., \& Heathcote, A. (2003). QMLE: Fast, robust, and efficient estimation of distribution functions based on quantiles. $B e-$ havior Research Methods, Instruments, \& Computers, 35, 485-492.

CHENG, R. C. H., \& AMIN, N. A. K. (1983). Estimating parameters in continuous univariate distributions with a shifted origin. Journal of the Royal Statistical Society: Series B, 45, 394-403.

Cheng, R. C. H., \& ILEs, T. C. (1987). Corrected maximum likelihood in non-regular problems. Journal of the Royal Statistical Society: Series B, 49, 95-101.

EкströM, M. (2001). Consistency of generalized maximum spacing estimates. Scandinavian Journal of Statistics, 28, 343-354.

Heathcote, A., Brown, S., \& Cousineau, D. (2004). QMPE: Estimating lognormal, Wald, and Weibull RT distributions with a parameter-dependent lower bound. Behavior Research Methods, Instruments, \& Computers, 36, 277-290.

Heathcote, A., Brown, S., \& Mewhort, D. J. K. (2002). Quantile maximum likelihood estimation of response time distributions. $P$ sychonomic Bulletin \& Review, 9, 394-401.

Hyndman, R. J., \& FAn, Y. (1996). Sample quantiles in statistical packages. American Statistician, 50, 361-365.

RANNEBY, B. (1984). The maximum spacing method: An estimation method related to the maximum likelihood method. Scandinavian Journal of Statistics, 11, 93-112.

Speckman, P. L., \& Rouder, J. N. (2004). A comment on Heathcote, Brown, and Mewhort's QMLE method for response time distributions. Psychonomic Bulletin \& Review, 11, 580-582.

TitTERington, D. M. (1985). Comment on "Estimating parameters in continuous univariate distributions." Journal of the Royal Statistical Society: Series B, 47, 115-116. 


\section{NOTES}

1. For example, Cheng and Amin (1983) showed that for the Gamma and Weibull distributions with shape parameter $<2$, the minimum parameter estimate has variance smaller than order $n^{-1}$, where $n$ is sample size.

2. For example, Heathcote et al.'s (2002) QML4 method, with exactly four observations falling between every two consecutive quantile estimates, uses $\hat{\mathbf{q}}=\left(-\infty,\left(x_{(4)}+x_{(5)}\right) / 2,\left(x_{(8)}+x_{(9)}\right) / 2, \ldots,\left(x_{(n-1)}+\right.\right.$ $\left.\left.x_{(n)}\right) / 2, \infty\right)$ and $m=(n-1) / 4$. In principle, QML may also be used with different quantile estimation algorithms (see Hyndman \& Fan, 1996) and uneven quantile intervals.

(Manuscript received July 14, 2003;

revision accepted for publication September 10, 2003.) 